Published in final edited form as:

Am J Med. 2016 June ; 129(6): 620-627. doi:10.1016/j.amjmed.2016.01.016.

\title{
Transient Hyponatremia during Hospitalization for Acute Heart Failure
}

\author{
Frederik H. Verbrugge, M.D. Ph.Da,b,c Justin L. Grodin, M.D. M.P.H. ${ }^{a}$, Mullens Wilfried, M.D. \\ Ph.D. ${ }^{b, d}$, David O. Taylor, M.D. ${ }^{a}$, Randall C. Starling, M.D. M.P.H. ${ }^{a}$, and W.H. Wilson Tang, \\ M.D. ${ }^{\mathrm{a}}$ \\ aDepartment of Cardiovascular Medicine, Heart and Vascular Institute, Cleveland Clinic, Ohio, \\ United States of America \\ bDepartment of Cardiology, Ziekenhuis Oost-Limburg, Genk, Belgium \\ 'Doctoral School for Medicine and Life Sciences, Hasselt University, Diepenbeek, Belgium \\ ${ }^{d B i o m e d i c a l ~ r e s e a r c h ~ i n s t i t u t e, ~ D e p a r t m e n t ~ o f ~ M e d i c i n e ~ a n d ~ L i f e ~ S c i e n c e s, ~ H a s s e l t ~ U n i v e r s i t y, ~}$ \\ Diepenbeek, Belgium
}

\begin{abstract}
Objective - To study whether the temporal pattern of transient hyponatremia development in acute heart failure might provide insight into its pathophysiology and prognostic relevance.

Methods-A post-hoc analysis of the ESCAPE and DOSE AHF studies was performed ( $n=716)$. Patients were stratified according to the temporal pattern of hyponatremia development: (1) no hyponatremia; (2) persistent hyponatremia; (3) decompensation hyponatremia disappearing with decongestive treatment; and (4) treatment-induced hyponatremia.
\end{abstract}

Results-Transient decompensation versus no hyponatremia was associated with significantly elevated blood urea nitrogen/creatinine ratio (P-value $<0.001)$, plasma renin activity ( $\mathrm{P}$ value $<0.001)$, and plasma aldosterone levels (P-value $<0.001)$ at baseline. Disease severity characteristics of such patients were intermediate between no and persistent hyponatremia. In contrast, patients with treatment-induced versus no hyponatremia had similar baseline characteristics, comparable natriuretic peptide levels, and both groups had little neurohumoral activation at baseline. Diuretic efficacy, defined as net fluid balance $[\mathrm{mL}]$ per $40 \mathrm{mg}$ furosemideequivalent dose administered, was lower in patients with persistent or treatment-induced hyponatremia versus decompensation hyponatremia or no hyponatremia, respectively. The former versus latter groups also had more pronounced neurohumoral activation with decongestive treatment. The risk for all-cause mortality $[\mathrm{HR}(95 \% \mathrm{CI})=2.50(1.50-4.19) ; \mathrm{P}$-value $<0.001]$ and death or heart failure readmission $[\mathrm{HR}(95 \% \mathrm{CI})=2.18(1.60-2.97)$; P-value $<0.001]$ was

Corresponding author: W.H. Wilson Tang MD, Department of Cardiovascular Medicine, Heart and Vascular Institute, Cleveland Clinic, 9500 Euclid Avenue, Desk J3-4, Cleveland, OH 44195, Phone: 216-444-2121 | Fax: 216-445-6165 |; Email: tangw@ ccf.org Conflict of interest: None. All authors had access to the data and a role in writing the manuscript.

Publisher's Disclaimer: This is a PDF file of an unedited manuscript that has been accepted for publication. As a service to our customers we are providing this early version of the manuscript. The manuscript will undergo copyediting, typesetting, and review of the resulting proof before it is published in its final citable form. Please note that during the production process errors may be discovered which could affect the content, and all legal disclaimers that apply to the journal pertain. 
significantly elevated in patients with persistent versus no hyponatremia, with the risk of decompensation and treatment-hyponatremia situated in-between.

Conclusions-Transient hyponatremia is prognostically relevant, but has a heterogeneous etiology according to its temporal pattern of development.

\section{Keywords}

sodium; heart failure; prognosis; diuretics

\section{Introduction}

Hyponatremia, defined as a serum sodium level $<135 \mathrm{mmol} / \mathrm{L}$, is common in heart failure. It is present in $\sim 20 \%$ of patients admitted with acute heart failure and develops during decongestive treatment in an additional $\sim 15-25 \%[1,2,3]$. Hyponatremia in heart failure is associated with worse outcomes and increased mortality, especially when persistent $[4,5]$. However, it remains unclear whether this relationship is causal or if hyponatremia just represents a marker of more advanced disease. Studies assessing the prognostic impact of correcting hyponatremia during decongestive treatment in acute heart failure have yielded conflicting results $[6,7]$. This should not be surprising as the underlying pathophysiological mechanisms of hyponatremia in heart failure are complex and diverse, warranting an individualized approach [8]. The temporal pattern of hyponatremia development might help to assess its etiology and could portend prognostic significance. The aim of the current analysis was to characterize two distinct patterns of transient hyponatremia development in patients with acute heart failure undergoing decongestive treatment: (1) decompensation hyponatremia, present upon admission but disappearing with decongestive treatment versus (2) treatment-induced hyponatremia, not present at admission but developed during decongestive treatment and present at discharge. Baseline and treatment characteristics as well as prognostic significance of those patterns were compared with both persistent and no hyponatremia development in patient populations from the Evaluation Study of Congestive Heart Failure and Pulmonary Artery Catheterization Effectiveness (ESCAPE) and Diuretic Optimization Strategies Evaluation in Acute Heart Failure (DOSE AHF) study.

\section{Material And Methods}

\section{Study design}

This study is a post-hoc analysis from the ESCAPE and DOSE AHF study. Detailed methods of those studies have been reported elsewhere [9, 10]. For the purpose of the current analysis, patients were selected who had serum sodium levels available at baseline and at least at one other moment during their index hospitalization. Patients not discharged alive were excluded. All-cause mortality, orthotopic heart transplantation, left ventricular assist device implantation, and heart failure readmissions were adjudicated end-points in both trials. 


\section{Hyponatremia patterns}

Persistent hyponatremia was defined as hyponatremia (i.e., serum sodium levels $<135$ $\mathrm{mmol} / \mathrm{L}$ ) present on admission and persisting till discharge. Decompensation hyponatremia was specified as admission hyponatremia that disappeared with decongestive treatment at discharge. Treatment-induced hyponatremia was defined by normal admission serum sodium levels (i.e., $\geq 135 \mathrm{mmol} / \mathrm{L}$ ) with a subsequent drop $<135 \mathrm{mmol} / \mathrm{L}$ at discharge. Baseline and treatment characteristics were compared according to the pattern of hyponatremia development. Plasma N-terminal of the prohormone of B-type natriuretic peptide (NTproBNP) levels, plasma renin activity and plasma aldosterone levels were assessed only in DOSE AHF patients with a full set of biomarkers available at baseline and after $72 \mathrm{~h}$ $(\mathrm{n}=308)$. For the same group, diuretic efficacy was defined as net fluid balance after $72 \mathrm{~h}$ per $40 \mathrm{mg}$ of furosemide-equivalent dose administered. Net fluid balance was not consistently reported in the ESCAPE.

\section{Study end-points}

The primary end-point for this analysis was all-cause mortality. The secondary end-point constituted the combination of all-cause mortality or unscheduled heart failure hospitalization. Data were censored in case of orthotopic heart transplantation or implantation of a left ventricular assist device or at the end of follow-up after 180 days in the ESCAPE and 60 days in the DOSE AHF study.

\section{Statistical analysis}

Continuous variables are expressed as mean \pm standard deviation if normally distributed or otherwise as median (interquartile range) and compared using the independent-samples Student's $t$-test, one-way analysis of variance, the Mann-Whitney $U$ test or the KruskalWallis $H$ test, as appropriate. Normality was assessed by the Shapiro-Wilk statistic. Categorical data are expressed as percentages and compared by Pearson's $X^{2}$-test. Cumulative, actuarial survival rates were calculated according to the Kaplan-Meier method with the log-rank test used for comparison among groups. Cox-proportional hazards models were used to calculate the hazard ratio (HR) with corresponding 95\% confidence interval ( $95 \%$ CI) for occurrence of the primary and secondary study end-points associated with each pattern of hyponatremia development. Statistical significance was always set at a 2-tailed probability level of $<0.05$. All statistics were performed using IBM ${ }^{\circ}$ SPSS ${ }^{\circledR}$ (version 22.0 for Windows). All authors had full access to the data and contributed to the writing of the manuscript. Together, they take responsibility for the integrity of the data and agree to the report as written.

\section{Results}

\section{Study population characteristics}

A study flowchart is presented in Figure 1. Seven hundred sixteen patients from the original ESCAPE and DOSE AHF studies (97\%) were included in the current analysis. Their baseline characteristics are presented in Table 1 . Twenty percent of patients presented with hyponatremia upon admission, with another 17\% developing it during decongestive 
treatment. Hyponatremia occurred more frequently in the ESCAPE (23\% and $21 \%$ for admission and treatment-induced hyponatremia, respectively) compared to the DOSE AHF study (15\% and $12 \%$, respectively). Development of hyponatremia at any time-point was associated with a lower left ventricular ejection fraction (Table 1). Persistent and decompensation but not treatment-induced hyponatremia patients demonstrated significantly elevated blood urea nitrogen (BUN) at baseline, despite similar serum creatinine levels compared to patients without hyponatremia (Table 1). In addition, patients with persistent compared to no hyponatremia also had significantly lower arterial blood pressure, with values in patients with decompensation or treatment-induced hyponatremia situated in between (Table 1).

\section{Decongestive treatment strategy and hyponatremia patterns}

None of the patterns of hyponatremia development were more common with either a strategy of continuous versus bolus or high-dose versus low-dose loop diuretics in the DOSE AHF study. There was also no association between hyponatremia development and randomization to pulmonary artery catheter-guided therapy versus usual care in the ESCAPE. The total dose of loop diuretics administered (i.e., use according to study protocol plus open label use) was $638 \mathrm{mg}$ (417-907 mg), $802 \mathrm{mg}$ (420-1,359 mg), $672 \mathrm{mg}$ (442-931 $\mathrm{mg}$ ), and $671 \mathrm{mg}(472-903 \mathrm{mg}$ ) furosemide equivalents in patients developing no hyponatremia, persistent hyponatremia, reversible decompensation hyponatremia and treatment-induced hyponatremia, respectively $(\mathrm{P}$-value $=0.207)$. Thiazide-type diuretics were used in 3\%,7\%,4\%, and 4\% among the same groups respectively (P-value $=0.404$ ).

\section{Diuretic efficacy and hyponatremia patterns in the DOSE AHF study}

Diuretic efficacy, defined as net fluid balance after $72 \mathrm{~h}$ per $40 \mathrm{mg}$ of furosemide-equivalent dose administered was similar in DOSE AHF patients without hyponatremia versus patients with reversible decompensation hyponatremia [277 mL $(159-466 \mathrm{~mL})$ versus $250 \mathrm{~mL}$ (202-399 mL); P-value $=0.862]$. In contrast, diuretic efficacy was significantly lower in patients with persisting hyponatremia $[172 \mathrm{~mL}(80-300 \mathrm{~mL})$; P-value $=0.043]$ and also nonsignificantly lower among patients with treatment-induced hyponatremia [168 $\mathrm{mL}(112-510$ $\mathrm{mL}$ ); P-value=0.540].

\section{Effective decongestion and hyponatremia patterns in the ESCAPE and DOSE AHF study}

In the DOSE AHF study, baseline levels of plasma NT-proBNP were not different at 4,435 $\mathrm{ng} / \mathrm{L}(2,446-9,907 \mathrm{ng} / \mathrm{L})$ regardless of the pattern of hyponatremia development (Pvalue $=0.359$; Table 2). Figure 2 shows the relative change in plasma NT-proBNP levels after decongestive treatment at discharge, which was significantly different according to the pattern of hyponatremia development (P-value $=0.010)$. While patients with treatmentinduced hyponatremia had a similar decrease in plasma NT-proBNP levels at discharge compared to patients without hyponatremia, this decrease was significantly less pronounced in patients with persistent hyponatremia and reversible decompensation hyponatremia (Table 2). Among 172 patients from the ESCAPE randomized to pulmonary artery catheter use, central venous pressure at discharge was $8 \pm 4 \mathrm{mmHg}$ in patients without hyponatremia, which was not different in patients with treatment-induced hyponatremia $(9 \pm 6 \mathrm{mmHg}$; P- 
value $=0.792$ ), but lower compared to patients with persistent or reversible decompensation hyponatremia (both $11 \pm 6 \mathrm{mmHg}$; P-value=0.019).

\section{Neurohumoral activation and hyponatremia patterns in the DOSE AHF study}

Table 2 also displays the baseline and discharge values for plasma renin activity and plasma aldosterone levels in the DOSE AHF study. The baseline levels of plasma renin activity, obtained before randomization, but often after the first administration of loop diuretics, were markedly elevated in patients with persistent hyponatremia (P-value $<0.001)$, intermediate in patients with decompensation hyponatremia $(\mathrm{P}$-value $=0.002)$, but not different in patient with treatment-induced hyponatremia $(\mathrm{P}$-value $=0.073)$ compared to patients without hyponatremia. After decongestive treatment however, the relative increase in plasma renin activity was the highest in the latter group. As a result, only patients with persistent (Pvalue $<0.001)$ and treatment-induced hyponatremia $(\mathrm{P}-$ value $=0.014)$ but not reversible decompensation hyponatremia $(\mathrm{P}$-value $=0.584)$ had significantly higher levels at discharge compared to patients without hyponatremia.

\section{Hyponatremia patterns and clinical outcome}

During follow-up, 96 patients died (13\%) and 239 were readmitted for heart failure (33\%).

Fourteen patients underwent placement of a left ventricular assist device and were censored at that time-point, with 6 of these patients subsequently undergoing orthotopic heart transplantation. Twenty-seven other patients were censored because of orthotopic heart transplantation. Only 19 patients (3\%) were censored because of loss to follow-up before the end of the study. Both the risk for all-cause mortality (Figure 3) and the risk for death or heart failure readmission (Figure 4) were significantly associated with the pattern of hyponatremia development that was observed. Patients who demonstrated persistent hyponatremia had the highest risk for both end-points (Table 3). The risk for adverse clinical outcome with reversible decompensation hyponatremia and treatment-induced hyponatremia was intermediate, while those without any hyponatremia had the lowest risk (Figure 3, 4).

\section{Discussion}

In this study, two distinct phenotypes of transient hyponatremia were identified. Patients with transient decompensation hyponatremia resolving with decongestive treatment had significantly elevated neurohumoral activation at baseline. Yet, diuretic efficacy was preserved in this group without further exacerbation of neurohumoral activation.

Nevertheless, the degree of decongestion achieved was similarly poor compared to patients with persistent hyponatremia, who represented the sickest group. The risk for adverse clinical outcomes in patients with resolving hyponatremia was intermediate between that of patients with persistent and no hyponatremia. In contrast, treatment-induced hyponatremia patients presented with similar natriuretic peptide levels upon admission compared to patients without hyponatremia development, with both groups demonstrating little neurohumoral activation. Despite similar and successful decongestion according to natriuretic peptide level changes in the DOSE AHF study and invasive hemodynamic measurements in the ESCAPE, the former group demonstrated poor diuretic efficacy and marked neurohumoral activation during treatment, suggesting that decongestive therapy was 
overly aggressive. Treatment-induced hyponatremia was common with an incidence of $17 \%$ and associated with a significant $43 \%$ relative risk increase for death or heart failure readmission.

Studies have consistently reported that persistent hyponatremia in heart failure characterizes a population that has more advanced disease, with more pronounced neurohumoral activation, and higher risk of adverse clinical outcomes, which is corroborated by the current analysis $[6,11,12]$. As the DOSE AHF data uniquely provide prospectively collected plasma renin activity and plasma aldosterone levels at baseline and discharge, it was shown that persistent hyponatremia in acute heart failure was associated with exceedingly high plasma renin activity levels during decongestive treatment, even in the contemporary area of neurohumoral blocker treatment. This was further supported by a high BUN/creatinine ratio in this group, which has been demonstrated to be among the strongest predictors of mortality in acute heart failure [13]. Clearly, sodium homeostasis in this group is markedly altered.

The current analysis further suggests that when hyponatremia is corrected by decongestive treatment, clinical outcome is better compared to patients with persistent hyponatremia, but still worse compared to patients without hyponatremia development. There are several potential explanations for this observation. First, arterial blood pressure and baseline neurohumoral activation suggested that patients with disappearing hyponatremia might have had less advanced heart failure compared to patients with persistent, but more advanced than patients with no hyponatremia. This argument is further reinforced by the results of Lee et al. who found that disappearing hyponatremia in acute heart failure was associated with adverse outcome, but the effect was no longer significant when corrected for markers of disease severity [7]. Intriguingly in the current analysis however, patients with decompensation hyponatremia disappearing with decongestive treatment also demonstrated no increase in neurohumoral activation during admission and showed preserved diuretic efficacy similar to patients without hyponatremia. Despite these seemingly favorable characteristics, decongestion achieved was poor, both assessed by natriuretic peptide level changes and invasively measured central venous pressure. The reason for this observation remains unclear, but it might well be that clinicians were reluctant to pursue thorough decongestion in patients perceived to be at high risk because of initial presentation with hyponatremia, further encouraged by subsequent correction of the latter. In addition, despite the fact that patients with reversible decompensation hyponatremia had the longest inhospital stay, the cumulative dose of diuretics used in this group was relatively low, similar to patients without hyponatremia, further suggesting that decongestive treatment might not have been aggressive enough in this group. This reinforces the widely accepted view that complete decongestion should be pursued in every heart failure patient to prevent readmissions [14].

Arguably the most important finding of the current analysis was that treatment-induced hyponatremia was associated with a significantly elevated risk of death or heart failure readmission. At baseline, serum BUN levels, plasma renin activity and plasma aldosterone suggested no significantly increased neurohumoral activation in this group. Furthermore, plasma NT-proBNP levels as a surrogate for disease severity were also similar in this group compared to patients without hyponatremia development. Moreover, it was clearly shown 
that patients with treatment-induced hyponatremia achieved adequate decongestion. What was strikingly different however between both groups was the amount of neurohumoral activation during decongestive treatment and the poor diuretic efficacy of patients with treatment-induced hyponatremia, findings suggesting that decongestive treatment was overly aggressive in this group. Indeed, it has been shown that the natriuretic response to diuretic treatment diminishes with progressive decongestion [15]. These results support a paradigm shift in the treatment of acute heart failure, as not only achieving effective decongestion, but also the way to achieve it (i.e., with more or less neurohumoral activation) might be important to improve clinical outcome.

\section{Study limitations}

The current analysis should be interpreted in the light of some study limitations. First, this was a post-hoc analysis and therefore results should be considered exploratory and hypothesis-generating. Second, because of the different study designs of the ESCAPE and DOSE AHF study, some data were uniquely available in only one study population. Specifically, plasma NT-proBNP, plasma renin activity and plasma aldosterone were available for DOSE AHF but not ESCAPE patients, while invasive hemodynamic data were available only for the 172 ESCAPE patients included in the pulmonary artery catheter arm. In addition, the study population characteristics of both trials were also different, with ESCAPE including advanced heart failure patients with reduced ejection fraction in contrast to DOSE AHF, which included both patients with reduced and preserved ejection fraction. However, data from both studies were complimentary and findings were congruent. Third, in a minority of cases, discharge serum sodium levels were missing and the last observation was carried forward (mainly from day 5 or 7 after decongestive therapy). However, sensitivity analysis without these cases did not alter the study results. Fourth, only 54 patients presented with the pattern of decompensation hyponatremia disappearing with decongestive treatment. Therefore, the analysis might have been underpowered to demonstrate statistical significance in this group. Indeed, the risk for adverse clinical outcome did not reach statistical significance in this group, but the Kaplan-Meier curve suggested an intermediate risk in-between that of patients with persistent and no hyponatremia, respectively. Fifth, neurohumoral blocker treatment with angiotensinconverting enzyme inhibitors, angiotensin receptor blockers and mineralocorticoid receptor antagonists may influence plasma renin activity and plasma aldosterone levels. However, this effect may be expected to be similar at baseline versus follow-up, not explain the differential increase among hyponatremia groups.

\section{Conclusions}

Acute heart failure patients who present with hyponatremia characterize a population with increased neurohumoral activation and more severe heart failure who have adverse clinical outcome. When neurohumoral activation is only moderate and diuretic efficacy preserved without further exacerbating neurohumoral activation, hyponatremia is often reversible with decongestive treatment and outcomes are relatively better in such cases, yet still worse compared to patients without hyponatremia development. In contrast, hyponatremia development during decongestive treatment depicts a population without significant 
neurohumoral activation at baseline and with very similar characteristics compared to patients without hyponatremia development. However, treatment-induced hyponatremia is associated with significant neurohumoral activation during decongestive treatment as well as lower diuretic efficacy. The exact reasons for this phenomenon are unclear but might be explained by sodium depletion rather than purely dilutional hyponatremia. This could have important therapeutic implications as treatment-induced hyponatremia patients, despite showing similar decongestion, had a lower event-free survival compared to patients without hyponatremia development. One might speculate that increased neurohumoral activation associated with treatment-induced hyponatremia contributed to this result. Therefore, further mechanistic studies should investigate whether strategies of slower decongestion, saline repletion, or combination treatment with vasodilator agents or non-loop diuretic agents are associated with less neurohumoral activation and a lower risk of treatment-induced hyponatremia.

\section{Acknowledgments}

None

Funding sources: Frederik Verbrugge is supported by a Ph.D. fellowship of the Research Foundation - Flanders (FWO, 11L8214N). Frederik Verbrugge and Wilfried Mullens are researchers for the Limburg Clinical Research Program (LCRP) UHasselt-ZOL-Jessa, supported by the foundation Limburg Sterk Merk (LSM), Hasselt University, Ziekenhuis Oost-Limburg and Jessa Hospital. Dr. Tang is supported by the National Institutes of Health grant R01HL103931.

\section{References}

1. Gheorghiade M, Abraham WT, Albert NM, et al. Relationship between admission serum sodium concentration and clinical outcomes in patients hospitalized for heart failure: an analysis from the OPTIMIZE-HF registry. Eur Heart J. 2007; 28:980-8. [PubMed: 17309900]

2. Konishi M, Haraguchi G, Ohigashi H, et al. Progression of hyponatremia is associated with increased cardiac mortality in patients hospitalized for acute decompensated heart failure. J Card Fail. 2012; 18:620-5. [PubMed: 22858077]

3. Shchekochikhin DY, Schrier RW, Lindenfeld J, et al. Outcome differences in community- versus hospital-acquired hyponatremia in patients with a diagnosis of heart failure. Circ Heart Fail. 2013; 6:379-86. [PubMed: 23512983]

4. Sato N, Gheorghiade M, Kajimoto K, et al. Hyponatremia and in-hospital mortality in patients admitted for heart failure (from the ATTEND registry). Am J Cardiol. 2013; 111:1019-25. [PubMed: 23312128]

5. Bavishi C, Ather S, Bambhroliya A, et al. Prognostic significance of hyponatremia among ambulatory patients with heart failure and preserved and reduced ejection fractions. Am J Cardiol. 2014; 113:1834-8. [PubMed: 24837261]

6. Madan VD, Novak E, Rich MW. Impact of change in serum sodium concentration on mortality in patients hospitalized with heart failure and hyponatremia. Circ Heart Fail. 2011; 4:637-43. [PubMed: 21673193]

7. Lee SE, Choi DJ, Yoon CH, et al. Improvement of hyponatraemia during hospitalisation for acute heart failure is not associated with improvement of prognosis: an analysis from the Korean Heart Failure (KorHF) registry. Heart. 2012; 98:1798-804. [PubMed: 23125248]

8. Verbrugge FH, Steels P, Grieten L, et al. Hyponatremia in acute decompensated heart failure: depletion versus dilution. J Am Coll Cardiol. 2015; 65:480-92. [PubMed: 25660927]

9. Shah MR, O'Connor CM, Sopko G, et al. Evaluation Study of Congestive Heart Failure and Pulmonary Artery Catheterization Effectiveness (ESCAPE): design and rationale. Am Heart J. 2001; 141:528-35. [PubMed: 11275915] 
10. Felker GM, Lee KL, Bull DA, et al. Diuretic strategies in patients with acute decompensated heart failure. N Engl J Med. 2011; 364:797-805. [PubMed: 21366472]

11. Schaer GL, Covit AB, Laragh JH, et al. Association of hyponatremia with increased renin activity in chronic congestive heart failure: impact of diuretic therapy. Am J Cardiol. 1983; 51:1635-8. [PubMed: 6344606]

12. Gheorghiade M, Rossi JS, Cotts W, et al. Characterization and prognostic value of persistent hyponatremia in patients with severe heart failure in the ESCAPE Trial. Arch Intern Med. 2007; 167:1998-2005. [PubMed: 17923601]

13. Fonarow GC, Adams KF Jr, Abraham WT, et al. Risk stratification for in-hospital mortality in acutely decompensated heart failure: classification and regression tree analysis. JAMA. 2005; 293:572-80. [PubMed: 15687312]

14. Gheorghiade M, Shin DD, Thomas TO, et al. Congestion is an important diagnostic and therapeutic target in heart failure. Reviews in cardiovascular medicine. 2006; 7 Suppl 1:S12-24. [PubMed: 16955056]

15. Verbrugge FH, Nijst P, Dupont M, et al. Urinary composition during decongestive treatment in heart failure with reduced ejection fraction. Circ Heart Fail. 2014; 7:766-72. [PubMed: 25037309] 


\section{Clinical Significance}

- The temporal pattern of hyponatremia development in patients with acute heart failure provides differential prognostic information and characterizes different phenotypes of patients regarding efficacy of decongestion and associated neurohumoral activation.

- In transient decompensation hyponatremia, decongestion was inadequate despite good diuretic efficacy.

- In contrast, treatment-induced hyponatremia was associated with good decongestion, marked neurohumoral activation and poor diuretic efficacy.

- Overdiuresis with marked neurohumoral activation and treatment induced hyponatremia should be avoided as it is associated with worse outcomes. 


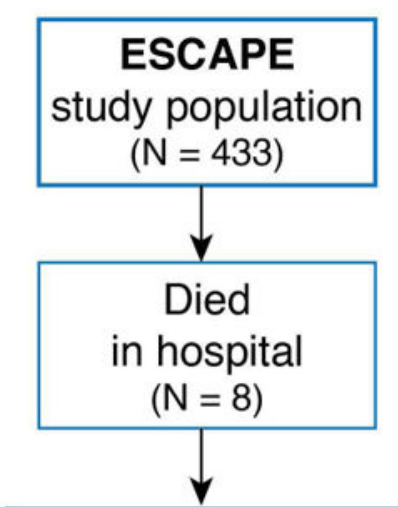

Missing baseline serum sodium value $(\mathrm{N}=3)$

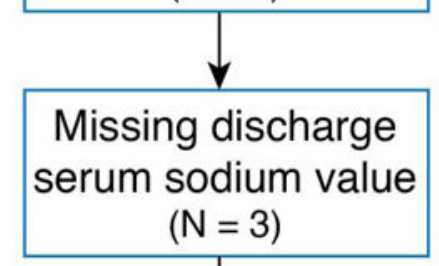
DOSE AHF study population $(\mathrm{N}=308)$

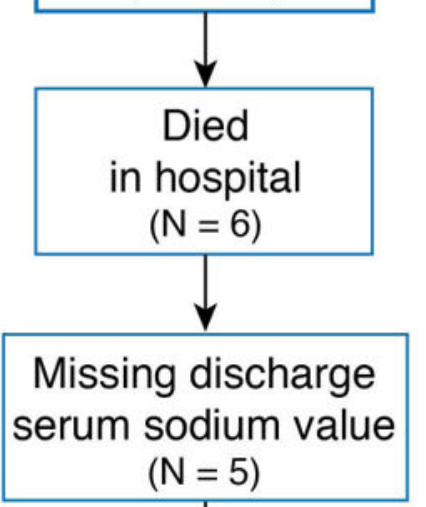

Study population $(\mathrm{N}=716)$

Figure 1.

Study flowchart. 


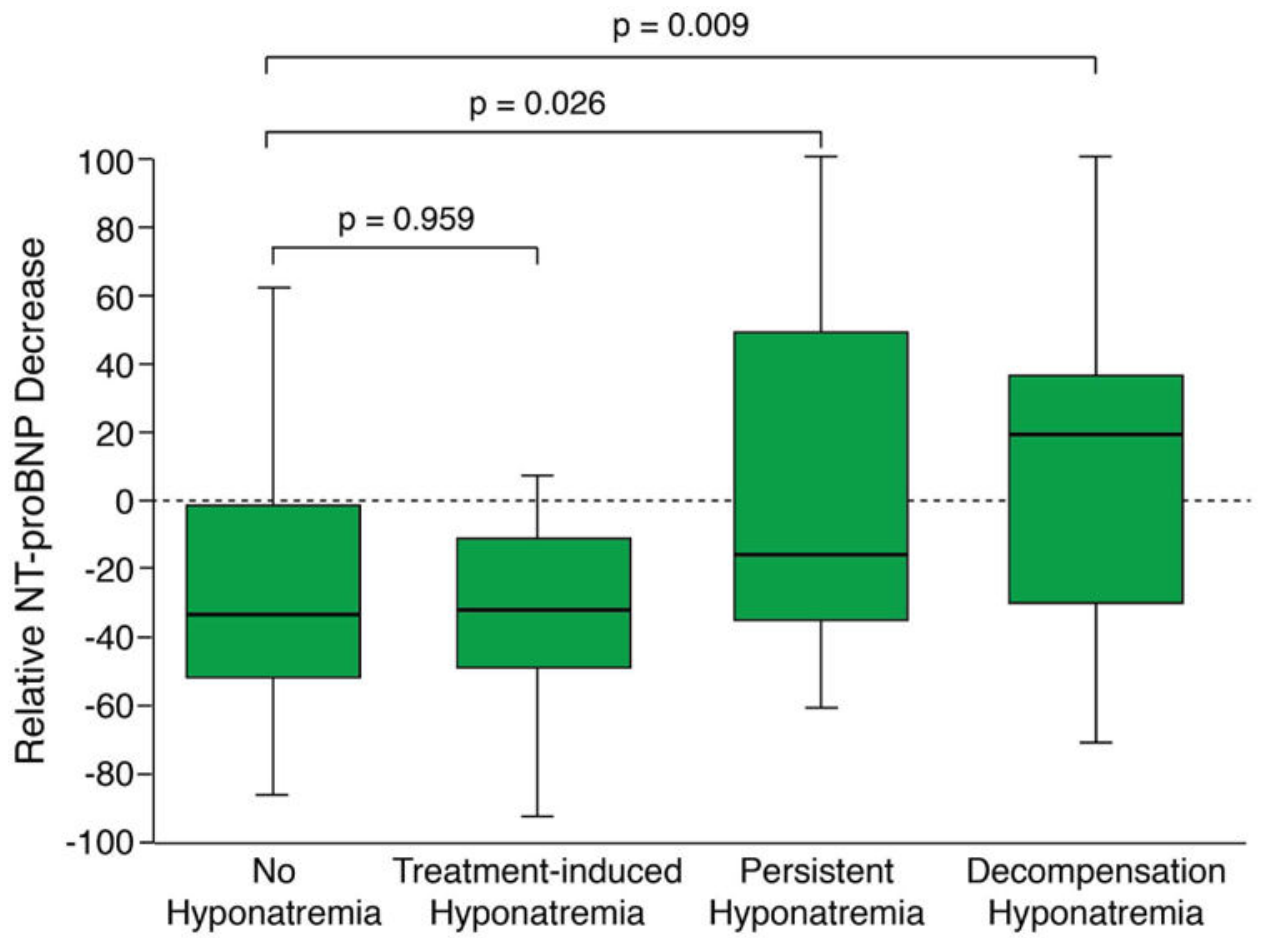

Figure 2.

Relative change in plasma NT-proBNP levels at discharge compared to baseline in the DOSE AHF study according to the pattern of hyponatremia development. NT-proBNP, Nterminal of the prohormone of B-type natriuretic peptide 


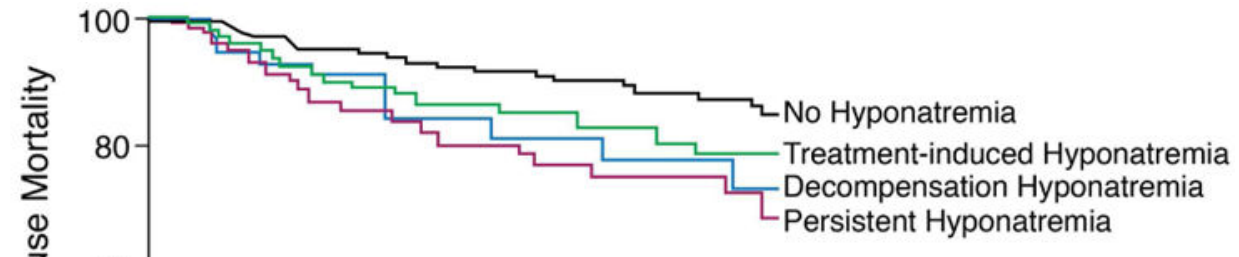

$\stackrel{1}{\overline{\frac{1}{4}}}$

혼

$40-$

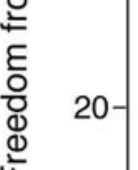

$0+$

030

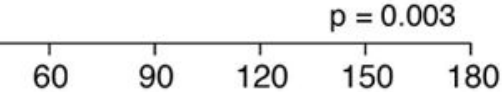

Patients at Risk:

\begin{tabular}{|c|c|c|c|c|c|c|}
\hline 450 & 430 & 350 & 196 & 186 & 182 & 118 \\
\hline 86 & 79 & 61 & 48 & 44 & 41 & 28 \\
\hline 53 & 50 & 42 & 25 & 23 & 22 & 15 \\
\hline 123 & 117 & 89 & 68 & 66 & 62 & 46 \\
\hline
\end{tabular}

Figure 3.

Freedom from all-cause mortality according to the pattern of hyponatremia development in the pooled data from the ESCAPE and DOSE AHF study. 


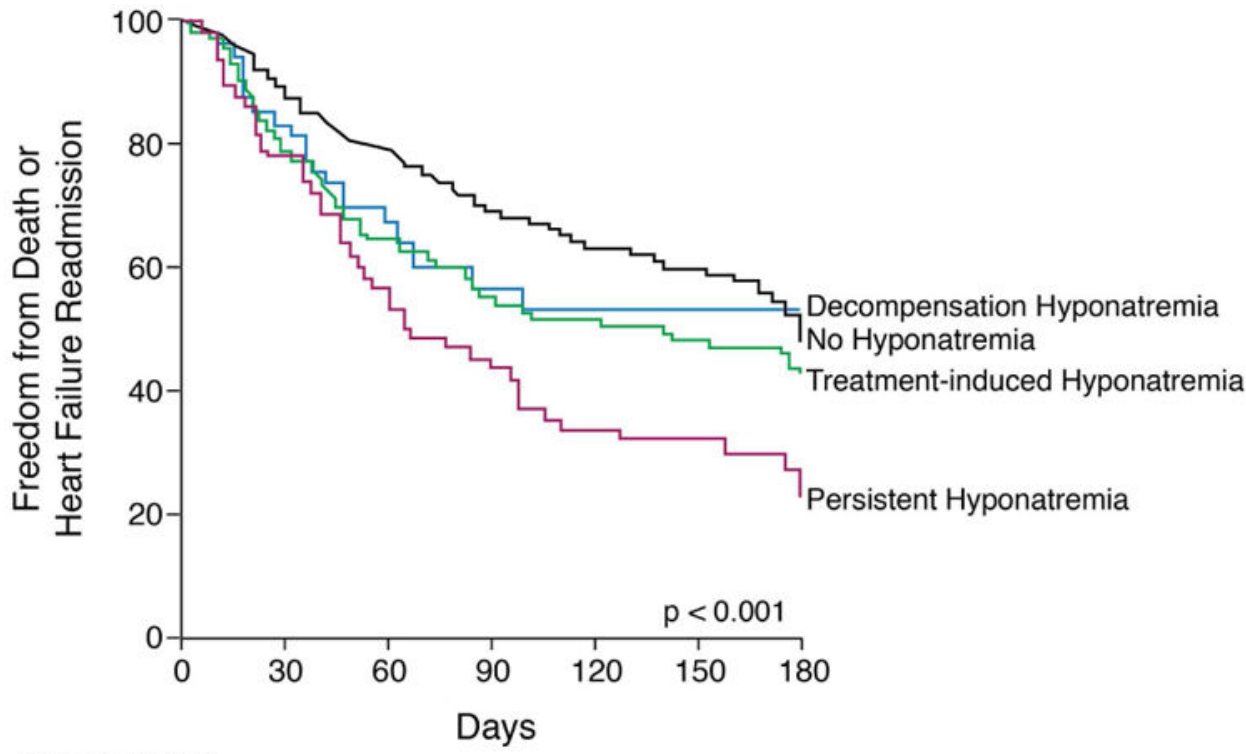

\begin{tabular}{|c|c|c|c|c|c|c|}
\hline itients at Risk: & & & & & & \\
\hline-450 & 393 & 296 & 144 & 128 & 118 & \\
\hline 86 & 64 & 40 & 27 & 19 & 18 & 11 \\
\hline 53 & 44 & 31 & 15 & 13 & 13 & \\
\hline 123 & 95 & 67 & 47 & 44 & 41 & \\
\hline
\end{tabular}

Figure 4.

Freedom from death or heart failure readmission according to the pattern of hyponatremia development in the pooled data from the ESCAPE and DOSE AHF study. 


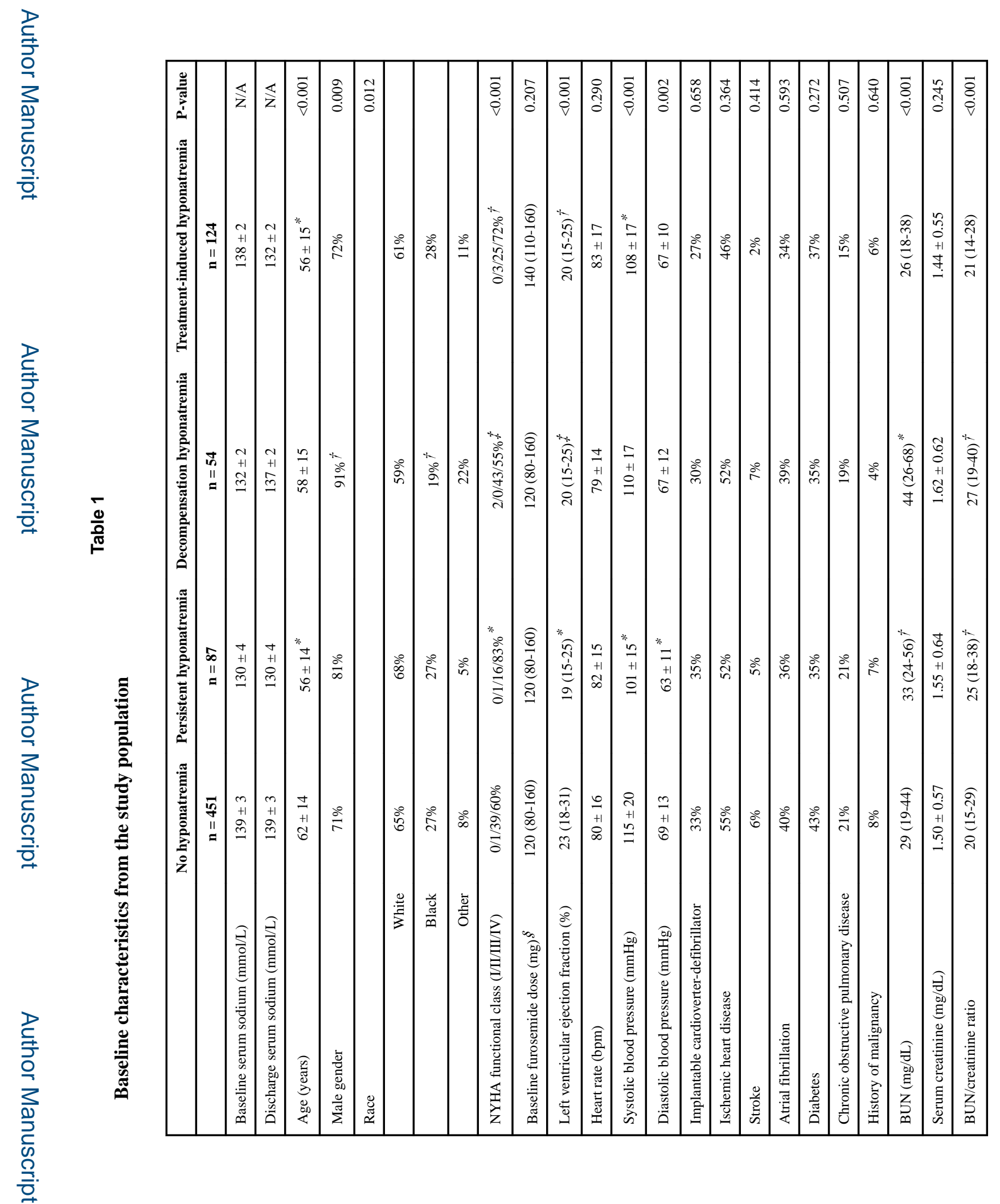

Am J Med. Author manuscript; available in PMC 2017 June 01. 

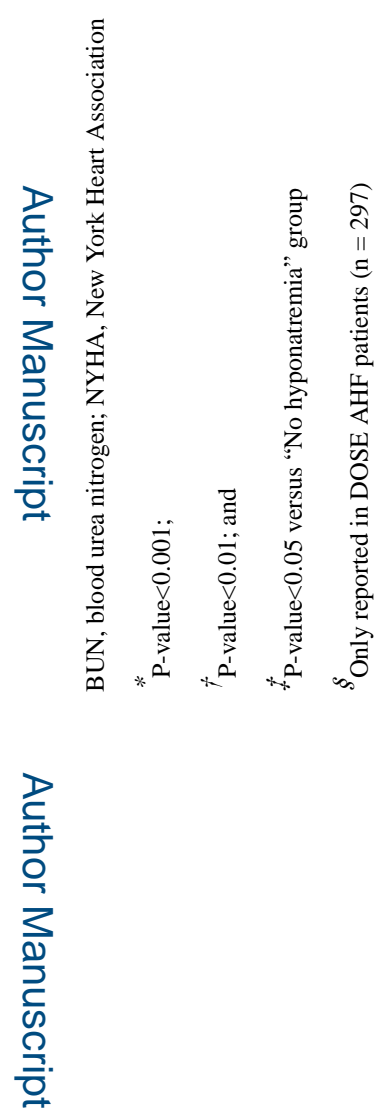

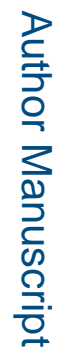

를

Am J Med. Author manuscript; available in PMC 2017 June 01. 


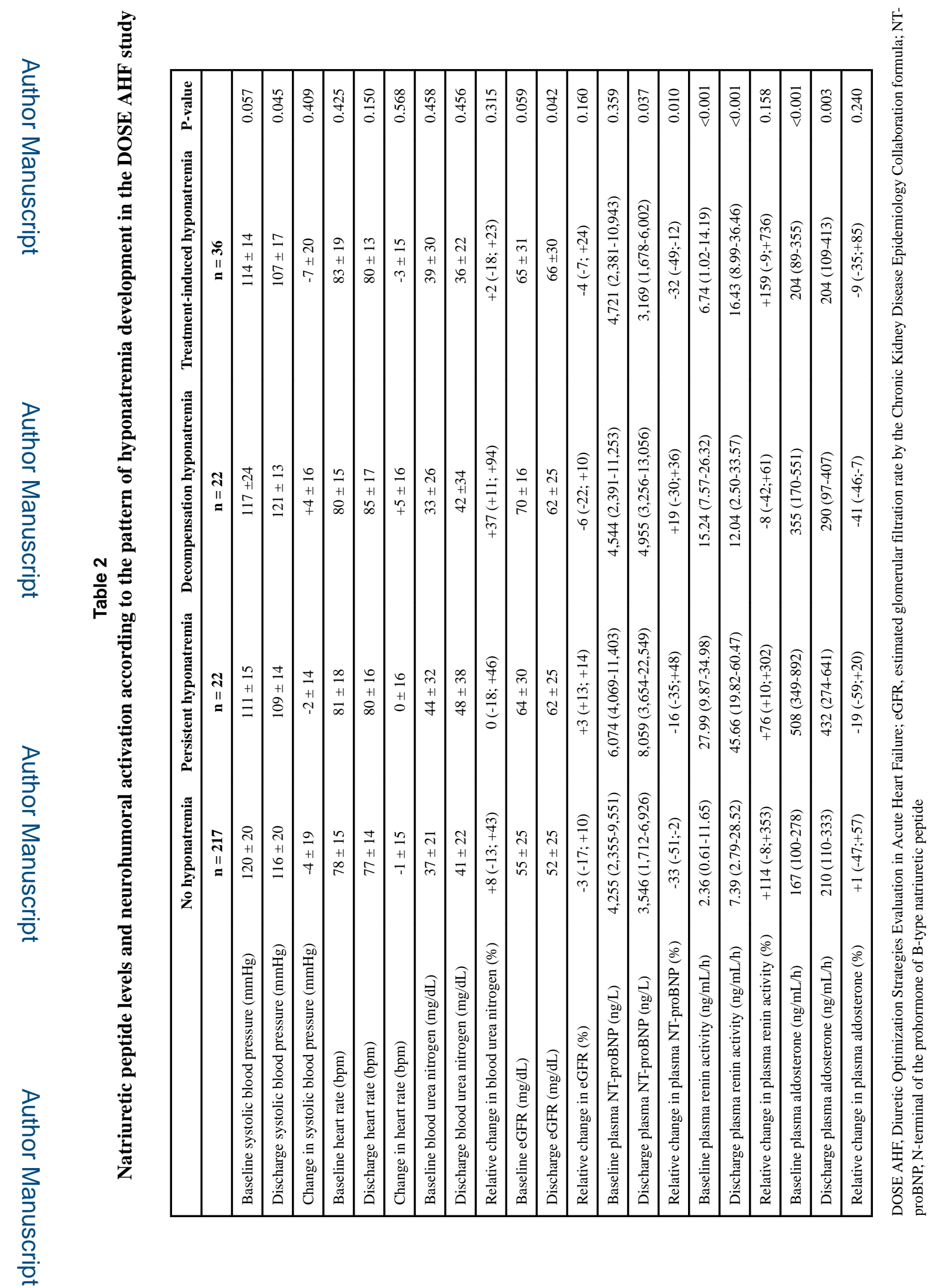

Am J Med. Author manuscript; available in PMC 2017 June 01. 
Table 3

Risk for adverse clinical outcome according to patterns of hyponatremia development

\begin{tabular}{|lcccc|}
\hline & \multicolumn{2}{c}{ All-cause mortality } & \multicolumn{2}{c|}{ Death or heart failure readmission } \\
\hline & HR $(\mathbf{9 5 \%} \mathbf{C I})$ & P-value & HR $(\mathbf{9 5 \%} \mathbf{C I})$ & P-value \\
\hline Persistent hyponatremia & $2.50(1.50-4.19)$ & $<0.001$ & $2.18(1.60-2.97)$ & $<0.001$ \\
\hline Decompensation hyponatremia & $1.93(0.97-3.85)$ & 0.060 & $1.26(0.80-1.98)$ & 0.327 \\
\hline Treatment-induced hyponatremia & $1.62(0.96-2.73)$ & 0.070 & $1.43(1.06-1.93)$ & 0.018 \\
\hline
\end{tabular}

